\title{
Theoretical Analysis of the Conformational Preference of the Phe-Phe-Phe Tripeptide Sequence
}

\author{
Masahito OKA, ${ }^{*}$ Yoshihiro BABA, Akihiro KaGEMOTO, \\ and Akio NAKAJIMA ${ }^{\dagger}$ \\ Departments of General Education and ${ }^{\dagger}$ Applied Chemistry, \\ Osaka Institute of Technology, Omiya, Asahi-ku, \\ Osaka 535, Japan
}

(Received June 2, 1989)

\begin{abstract}
To investigate local interactions in tripeptide sequences composed of amino acids having aromatic side chains, conformational analysis of $N$-acetyl- $N^{\prime}$-methylamide of the Phe-PhePhe tripeptide was theoretically carried out using the conformational energy-minimization procedure. The calculated results show that extended conformations are favorable conformations and $\beta$-bend and $\alpha$-helical conformations are unstable ones for the Phe-Phe-Phe sequence. These results accord with the previous results that extended conformation of the Tyr21-Phe22-Tyr23 sequence in BPTI is stabilized by short-range interaction and also that the tripeptide sequence composed of Phe and Tyr residues found in other proteins also have a tendency to take extended conformations. Experimental results on oligopeptides composed of the Phe residue in the solid state and solutions also support our calculated results. It was also shown that inter-residue interactions indicate minor effects on the mean-square end-to-end distance of a tripeptide.
\end{abstract}

KEY WORDS Conformational Energy / ECEPP / Phe-Phe-Phe /

Tripeptide / Extended Structure / $\beta$-Bend / Short-Range Interaction /

Protein /

The conformational state of each residue in protein is stabilized by short-, medium-, and long-rảnge interactions. Through analysis using minimization procedure of conformational energy for peptide sequence of BPTI (bovin pancreatic trypsin inhibitor), ${ }^{1}$ it was shown that extended conformations were stabilized by short- and long-range interactions. That is, the calculated lowest-energy conformation of half the residue situated in the extended region (Glu7-Tyr10, Ala16-Asn24, and Leu29-Tyr35) corresponds to the native conformation within short-range interactions, and that of another half residue does not correspond to the native conformation within short-and medium-range interactions. But, taking the effects of long-range interactions into consideration, the calculated lowest-energy conformation of all residues in extended region corresponds to the native conformation. The extended conformation of three residues (Tyr21, Phe22, and Tyr23) in BPTI was correctly predicted with short-range interactions, i.e., inter-residue interactions within tripeptide sequence. The sequence Tyr21-Phe22-Tyr23 was found in other homologous proteins ${ }^{2-4}$ mutated between Phe and Tyr residues with only one exception, that Tyr 21 was mutated by Ile in Russel's viper toxin. ${ }^{2}$ It was supposed that these kinds of mutations between Phe and Tyr residues at Tyr21-Phe22-Tyr23 caused no change in the three-dimensional structure of BPTI. $^{2}$

In the previous analysis of tripeptide-

\footnotetext{
* To whom all correspondence should be addressed.
} 
sequence of BPTI, ${ }^{1}$ conformations of the first and third residues of a tripeptide were fixed to those of the refined crystal structure of BPTI, ${ }^{5}$ and only $\phi, \psi$, and $\chi$ of the second residue of the tripeptide were selected as variables in conformational-energy minimizations. That is, as conformations of $\mathrm{Arg} 20$ and Phe22 in Arg20-Tyr21-Phe22, Tyr21 and Tyr23 in Tyr21-Phe22-Tyr23, and Phe22 and Asn24 in Phe22-Tyr23-Asn24 were fixed to extended conformations, the conformational preference of the second residue of these tripeptides might be affected by the restricted conformation of the nearest-neighbor residues. It is very interesting to elucidate the conformational preference of tripeptide sequences composed of Phe and Tyr residues, and also to evaluate the effects of short-range interactions in these sequence for stabilizing the extended conformation. In this work, conformational energy calculations were tried on the Phe-Phe-Phe tripeptide as a model of the tripeptide sequence composed of Phe and Tyr residues based on homologous properties between Phe and Tyr residues. ${ }^{2,6}$ Theoretical analyasis was carried out over the entire conformational space of the backbone and side chain.

Through theoretical analysis of poly( $\mathrm{L}$ phenylalanine) and poly(L-tyrosine $)^{6}$ based on intra-residue interactions, it was shown that extended conformations were most stable for Phe and Tyr residues with favorable intra-residue side-chain/backbone interactions, and also that the characteristic ratios of poly(L-phenylalanine) and poly(L-tyrosine) were larger than those of poly(L-alanine), ${ }^{6}$ poly(L-glutamine) and poly(L-glutamic acid), ${ }^{7}$ poly(L-leucine), ${ }^{8}$ and poly(L-valine). ${ }^{9}$ Good agreement between theoretical ${ }^{6,7}$ and experimental ${ }^{7,10-13}$ results indicate the validity of the treatments based on the intra-residue interactions and further demonstrates that the characteristic ratio can be decided by intraresidue interactions. However, it is supposed that aromatic residues such as Phe and Tyr, which present favorable intra-residue side-chain/backbone interactions, may also present favorable side-chain/side-chain interactions between adjacent residues. It is thus interesting to investigate how characteristic ratios of polypeptides with aromatic side chain are affected by the side-chain/side-chain interactions. To investigate the effects of these interactions within a tripeptide on the mean-square end-to-end distance, the statistically averaged square of the end-to-end distance was calculated using the results of energy minimizations of Ac-Phe-Phe-Phe$\mathrm{NHMe}$ and compared with the mean-square end-to-end distance evaluated by the statistically averaged transformation matrix $\langle\mathrm{T}\rangle$ of Phe residue. ${ }^{6}$

\section{THEORETICAL}

The nomenclature and conventions adopted are those recomended by IUPAC-IUB nomenclature commission. ${ }^{14}$ Conformational energy calculations of the Ac-Phe-Phe-Phe$\mathrm{NHMe}$ tripeptide were carried out with ECEPP (Empirical Conformational Energy Program for Peptides).$^{15}$ Energy minimization was carried out with the Powell algorithm ${ }^{16}$ until conformational energy did not change more than $0.001 \mathrm{kcal} \mathrm{mol}^{-1}$ between successive iterations. During minimizations, all $\phi, \psi, \chi^{1}$, and $\chi^{2}$ were allowed to vary. All other dihedral angles were held fixed at $180^{\circ}$. All combinations of single-residue minima ${ }^{17}$ of Phe were used as starting conformations (i.e., 3375 starting conformations).

The same definitions of bend and hydrogenbonded conformations in ref 18 were used. The classification of the bend type follows Table I of ref 18 . The relative conformational energy $\Delta E_{i}$, end-to-end distance $R$, and total bend probability $P_{\mathrm{b}}$ are defined in ref 18 . Conformational space was devided into 16 regions with the conformational letter codes shown in Figure 1 of ref 17. The statistical average of the conformation-dependent property $\mathbf{A}$ for the ensemble is defined by eq 3 of 
Table I. Calculated minimum energy conformations ${ }^{\mathrm{a}}$ of Ac-Phe-Phe-Phe-NHMe

\begin{tabular}{|c|c|c|c|c|c|c|c|c|c|}
\hline $\begin{array}{l}\text { Conformational } \\
\text { letter code }\end{array}$ & $\frac{\Delta E^{\mathrm{b}}}{\mathrm{kcal} \mathrm{mol}^{-1}}$ & $v^{\mathrm{c}}$ & $\frac{R}{\AA}$ & $\phi_{\text {Phe1 }}$ & $\psi_{\text {Phe1 }}$ & $\phi_{\text {Phe2 }}$ & $\psi_{\mathrm{Phe} 2}$ & $\phi_{\mathrm{Phe} 3}$ & $\psi_{\mathrm{Phe} 3}$ \\
\hline $\mathrm{ECC}$ & 0.00 & 0.129 & 12.35 & -156 & 149 & -81 & 101 & -86 & 74 \\
\hline $\mathrm{CDE}$ & 0.16 & 0.099 & 12.81 & -87 & 125 & -144 & 64 & -144 & 155 \\
\hline ECC & 0.21 & 0.091 & 11.95 & -158 & 165 & -61 & 109 & -83 & 76 \\
\hline CDD & 0.31 & 0.077 & 11.39 & -87 & 128 & -143 & 53 & -141 & 46 \\
\hline $\mathrm{EDE}$ & 0.55 & 0.052 & 12.28 & -151 & 113 & -145 & 47 & -157 & 159 \\
\hline $\mathrm{ECA}$ & 0.68 & 0.041 & 11.79 & -157 & 166 & -64 & 106 & -79 & -33 \\
\hline $\mathrm{FCC}$ & 0.69 & 0.041 & 11.87 & -74 & 143 & -82 & 103 & -87 & 74 \\
\hline CDD & 0.73 & 0.038 & 11.15 & -85 & 113 & -128 & 53 & -141 & 47 \\
\hline EFE & 0.74 & 0.037 & 13.49 & -155 & 152 & -78 & 152 & -141 & 152 \\
\hline $\mathrm{ECA}$ & 0.79 & 0.035 & 11.91 & -156 & 147 & -80 & 101 & -81 & -29 \\
\hline EDC & 0.91 & 0.028 & 11.82 & -153 & 134 & -151 & 31 & -109 & 60 \\
\hline $\mathrm{ECF}$ & 0.94 & 0.027 & 12.28 & -157 & 163 & -63 & 110 & -82 & 148 \\
\hline EFE & 1.10 & 0.021 & 13.57 & -155 & 152 & -80 & 156 & -145 & 152 \\
\hline EDF & 1.12 & 0.020 & 12.85 & -153 & 133 & -154 & 35 & -112 & 156 \\
\hline FDC & 1.20 & 0.017 & 11.73 & -100 & 132 & -149 & 30 & -107 & 65 \\
\hline EEE & 1.24 & 0.016 & 14.23 & -156 & 153 & -147 & 154 & -154 & 159 \\
\hline FCA & 1.36 & 0.013 & 11.26 & -72 & 141 & -81 & 104 & -83 & -29 \\
\hline FDE & 1.38 & 0.013 & 12.70 & -102 & 131 & -152 & 33 & -110 & 154 \\
\hline FFE & 1.44 & 0.012 & 13.04 & -73 & 138 & -79 & 153 & -129 & 147 \\
\hline ECC & 1.48 & 0.011 & 12.26 & -156 & 154 & -81 & 91 & -81 & 82 \\
\hline EEC & 1.52 & 0.010 & 12.20 & -157 & 150 & -154 & 150 & -83 & 85 \\
\hline $\mathrm{CDE}$ & 1.55 & 0.010 & 12.07 & -105 & 120 & -153 & 35 & -152 & 157 \\
\hline ECF & 1.61 & 0.009 & 12.50 & -157 & 154 & -80 & 86 & -74 & 144 \\
\hline EDE & 1.62 & 0.009 & 12.18 & -150 & 123 & -155 & 37 & -153 & 157 \\
\hline FEE & 1.64 & 0.008 & 13.74 & -86 & 149 & -144 & 153 & -159 & 158 \\
\hline EEE & 1.67 & 0.008 & 14.22 & -155 & 151 & -143 & 154 & -159 & 158 \\
\hline EFE & 1.69 & 0.008 & 13.61 & -156 & 141 & -96 & 146 & -159 & 159 \\
\hline $\mathrm{CCG}$ & 1.80 & 0.006 & 10.44 & -83 & 77 & -84 & 76 & -149 & -53 \\
\hline FFE & 1.84 & 0.006 & 13.05 & -79 & 148 & -76 & 157 & -145 & 152 \\
\hline EFE & 1.90 & 0.005 & 13.49 & -157 & 156 & -93 & 147 & -158 & 159 \\
\hline AAA & 1.91 & 0.005 & 6.00 & -69 & -31 & -61 & -40 & -83 & -39 \\
\hline EEE & 1.96 & 0.005 & 14.23 & -158 & 161 & -156 & 149 & -146 & 154 \\
\hline EEE & 2.00 & 0.005 & 14.26 & -156 & 155 & -149 & 158 & -159 & 158 \\
\hline
\end{tabular}

a All minima with $\Delta E<2 \mathrm{kcal} \mathrm{mol}^{-1}$.

b $E_{0}=-10.58 \mathrm{kcal} \mathrm{mol}^{-1}$.

c Values at $300 \mathrm{~K}$.

ref 19 . The averaged second moment of the end-to-end distance of tripeptide (the distance between $\mathrm{C}$-atom of acetyl group and that of NHMe group) based on the intra-residue interactions was calculated by the following equation. $^{20}$

$$
\begin{gathered}
\left\langle\mathrm{R}^{2}\right\rangle_{\text {intra }}=l^{2}\left[4\left(E_{3}+\langle\mathrm{T}\rangle\right)\left(E_{3}-\langle\mathrm{T}\rangle\right)^{-1}\right. \\
\left.-2\langle\mathrm{~T}\rangle\left(E_{3}-\langle\mathrm{T}\rangle^{4}\right)\left(E_{3}-\langle\mathrm{T}\rangle\right)^{-2}\right]_{11}
\end{gathered}
$$

where, $E_{3}$ is $3 \times 3$ unit matrix, the subscript 11 denotes that the 1,1 -element of matrix, $l$ is the virtual bond length, respectively. The statistically averaged transformation matrix $\langle\mathrm{T}\rangle$ of Phe was obtained in ref 6 taking average over the whole $\left(\phi, \psi, \chi^{1}, \chi^{2}\right)$ space for the $15^{\circ}$ interval.

\section{RESULTS AND DISCUSSION}

All minimum energy conformations of Ac- 

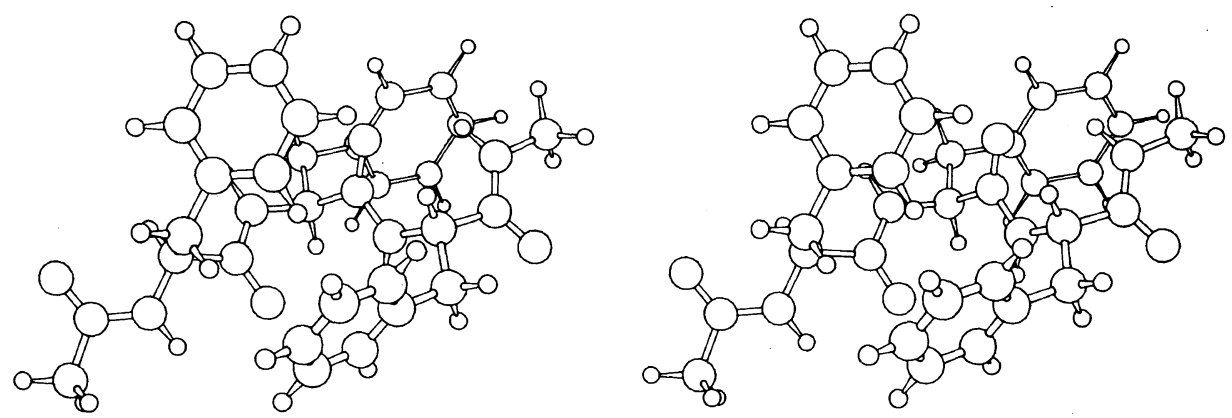

(a)
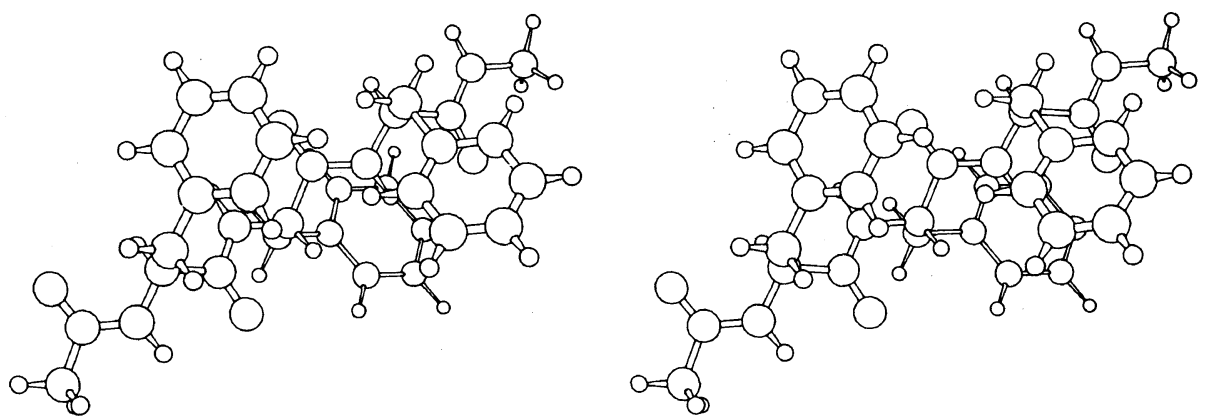

(b)
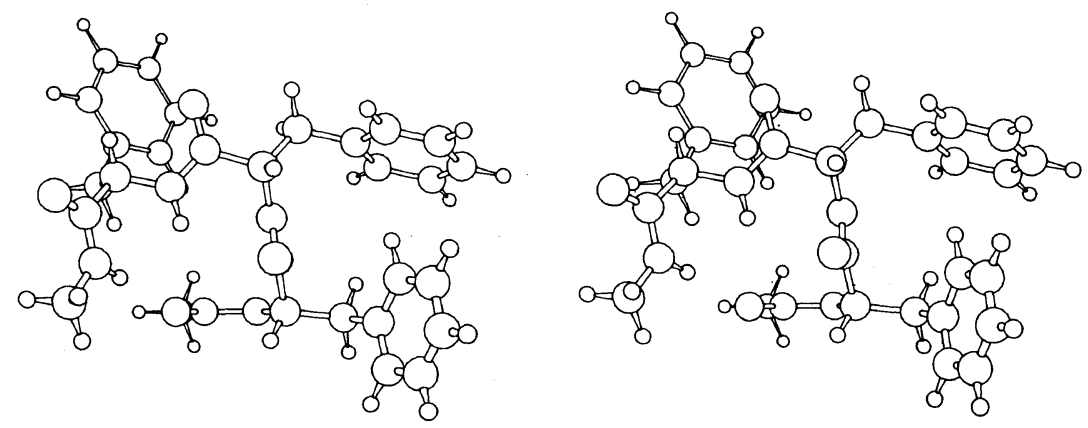

(c)

Figure 1. Minimum energy conformations of Ac-Phe-Phe-Phe-NHMe.

(a) Lowest-energy conformation with $\Delta E=0.00 \mathrm{kcal} \mathrm{mol}^{-1}$. The $\phi$ and $\psi$ of the three residues are $\left(-156^{\circ}\right.$, $\left.149^{\circ},-81^{\circ}, 101^{\circ},-86^{\circ}, 74^{\circ}\right)$ and the corresponding conformational letter code is ECC.

(b) 16th low-energy conformation with $\Delta E=1.24 \mathrm{kcal} \mathrm{mol}^{-1}$. The $\phi$ and $\psi$ of the three residues are $\left(-156^{\circ}, 153^{\circ},-147^{\circ}, 154^{\circ},-154^{\circ}, 159^{\circ}\right)$ and the corresponding conformational letter code is EEE.

(c) 79 th low-energy conformation with $\Delta E=2.95 \mathrm{kcal} \mathrm{mol}^{-1}$. The $\phi$ and $\psi$ of the three residues are $\left(-68^{\circ}\right.$, $-34^{\circ},-102^{\circ}, 40^{\circ},-152^{\circ},-54^{\circ}$ ) and the corresponding conformational letter code is ABG.

Phe-Phe-Phe-NHMe with $\Delta E<2 \mathrm{kcal} \mathrm{mol}^{-1}$ are listed in Table I (Phe1, Phe2, and Phe 3 are abbreviated as the first, second and third residues of this tripeptide). The lowest-energy conformation is the extended conformation with the letter code ECC and $\left(\phi_{1}, \psi_{1}, \phi_{2}, \psi_{2}\right.$, $\left.\phi_{3}, \psi_{3}\right)=\left(-156^{\circ}, 149^{\circ},-81^{\circ}, 101^{\circ},-86^{\circ}\right.$, $\left.74^{\circ}\right)$ as shown in Figure 1a. The same EC 
conformation at Phe1-Phe2 of a tripeptide was found as the 6th conformation of Ac-PhePhe-NHMe (M. Oka and A. Nakajima, unpublished data). However, the same CC conformation at Phe2-Phe3 of tripeptide was not found as a stable conformation of Ac-PhePhe-NHMe with $\Delta E<10 \mathrm{kcal} \mathrm{mol}^{-1}$. An analogous CC conformation, which has a different value of $\chi_{1}^{1}\left(=-62^{\circ}\right)$, was found as the 28 th conformation of Ac-Phe-Phe-NHMe with $\Delta E=2.10 \mathrm{kcal} \mathrm{mol}^{-1}\left(\chi_{2}^{1}=178^{\circ}\right.$ for Ac-PhePhe-Phe-NHMe). The ECC conformation also appeared as the 3rd conformation which has almost the same dihedral angles as the lowest-energy ECC conformation with one exception $\chi^{1}{ }_{1}=63^{\circ}\left(\chi^{1}{ }_{1}=178^{\circ}\right.$ for the lowestenergy ECC conformation). The above two ECC conformations each have a (Phe2)CO .. HN(NHMe) hydrogen bond. This hydrogen bond is a common type for the $\mathrm{C}$ conformation. ${ }^{17}$ The CC conformation usually has two hydrogen bonds of this type. ${ }^{21,22}$ However, the third and most stable ECC conformations lack the (Phel)CO $\cdots \mathrm{HN}(\mathrm{Phe} 3)$ hydrogen bond because of small deviation of the backbone conformation of the Phe 2 residue due to interactions between the side chains of Phel and Phe 3 residues. A typical extended conformation with the letter code EEE was found as the 16th conformation with $\Delta E=1.24 \mathrm{kcal} \mathrm{mol}^{-1}$ as shown in Figure $1 \mathrm{~b}$. Three phenyl groups are alternatively situated on the opposite sides of the backbone of the tripeptide, but their orientation has incomplete regularity, i.e., $\chi_{1}^{1}$ is almost the same as to $\chi_{2}^{1}$, but not $\chi^{1}{ }_{3}$. Only one EEE conformation (35th conformation) having the regularity of the side-chain orientations was found with $\Delta E<3 \mathrm{kcal} \mathrm{mol}^{-1}$, but it is energetically unfavorable in comparison with four EEE conformations having irregularities of the side-chain orientations in Table I.

Only 7 bend conformations were found among 87 conformations with $\Delta E<3 \mathrm{kcal} \mathrm{mol}^{-1}$. All of them are bend at Phe1-Phe 2 and
Table II. Calculated conformation-dependent properties $^{a}$ of Ac-Phe-Phe-Phe-NHMe

\begin{tabular}{ccc}
\hline & $\begin{array}{c}\text { Ac-Phe-Phe- } \\
\text { NHMe }\end{array}$ & $\begin{array}{c}\text { Ac-Phe-Phe- } \\
\text { Phe-NHMe }\end{array}$ \\
\hline$P_{\mathrm{b}}$ & & \\
Phel-Phe2 & 0.040 & 0.013 \\
Phe2-Phe3 & - & 0.010 \\
$\langle\mathbf{R}\rangle_{\text {inter }}(\AA)$ & 9.84 & 12.25 \\
$\left\langle\mathbf{R}^{2}\right\rangle_{\text {inter }}(\AA)$ & 97.9 & 151.2 \\
$\left\langle\mathbf{R}^{2}\right\rangle_{\text {intra }}(\AA)$ & 94.8 & 157.4 \\
\hline
\end{tabular}

a Values at $300 \mathrm{~K}$.

b Unpublished data (M. Oka and A. Nakajima).

five of them are bend at Phe2-Phe3, and three of them are double-bend with type III-III which is a part of the $\alpha$-helical conformation. The typical double-bend conformation (79th conformation with $\Delta E=2.95 \mathrm{kcal}$ $\mathrm{mol}^{-1}$ ), which is stabilized by the hydrogen bond between (Ac)CO $\cdots \mathrm{HN}(\mathrm{NHMe})$, is shown in Figure 1c.

As shown by the large value of $R$ in Table $\mathrm{I}$, most of the stable conformations of Phe-PhePhe tripeptide sequence are extended conformations, that is the Phe-Phe-Phe tripeptide sequence has a tendency to form an extended conformation with intra- and inter-residue interactions of the tripeptide sequence. These properties are clearly different from the previous theoretical results for $\mathrm{X}-\mathrm{Pro}-\mathrm{Y}$ tripeptides. $^{23}$

A detailed comparison of the backbone conformations of each Phe residue in Ac-PhePhe-Phe-NHMe with those of Phe residue in Ac- $P$ he- $-\mathrm{NHMe}^{17}$ indicates that $\mathrm{C}$ and $\mathrm{D}$ conformations of the second and third residues and $F$ conformation of the first residue of tripeptide are stabilized by the inter-residue interactions, but that the $\mathrm{E}$ conformation of the second residue is destabilized by interresidue interactions. That is, the relative conformational stabilities of the residue in tripeptide are affected by inter-residue interactions. However, the mean-square end-to-end distance based on the intra-residue interac- 
Table III. Backbone conformations of tripeptide-sequence composed of Phe and Tyr residues in proteins

\begin{tabular}{lcccrrrrrr}
\hline Protein & Sequence & $\begin{array}{c}\text { Conformational } \\
\text { letter code }\end{array}$ & $\phi_{1}$ & $\psi_{1}$ & $\phi_{2}$ & $\psi_{2}$ & $\phi_{3}$ & $\psi_{3}$ \\
\hline Trypsin inhibitor & Tyr21 -Phe22 -Tyr23 & EEF & -117 & 135 & -138 & 134 & -73 & 141 \\
Insulin & Phe24 -Phe25-Tyr26 & EED & -155 & 171 & -128 & 139 & -124 & 105 \\
Thermolysin & Tyr27-Tyr28-Tyr29 & ECF & -147 & 150 & -89 & 98 & -92 & 153 \\
Subtilisin & Phe261-Tyr262-Tyr263 & BBA & -158 & -28 & -131 & -18 & -103 & -38 \\
\hline
\end{tabular}

tions $\left\langle\mathrm{R}^{2}\right\rangle_{\text {intra }}$ calculated with $\langle\mathrm{T}\rangle_{\text {Phe }}$ in ref 6 is almost in good agreement with that based on the intra- and inter-residue interactions $\left\langle\mathrm{R}^{2}\right\rangle_{\text {inter }}$. That is, the relative stabilities of each conformation of Phe residue are changed by inter-residue interactions, but conformationdependent properties, especially mean-square end-to-end distance, are not so affected by these inter-residue interactions as whole. This is also supported by the facts that the theoretically evaluated characteristic ratios of polypeptides based on the intra-residue interactions show good agreement with those experimentally evaluated. , $^{6}$

By sequence analysis of proteins whose three-dimensional structures are shown, three tripeptide sequences composed of Phe and Tyr residues are found in insulin (B-chain, Phe24-Phe25-Tyr26), thermolysin (Tyr27Tyr28-Tyr29), and subtilisin (Phe261-Tyr262Tyr263) in addition to Tyr21-Phe22-Tyr23 of BPTI. All the backbone conformations of these four tripeptide-sequences composed of Phe and Tyr residues are shown in Table III. The conformations of Tyr21-Phe22-Tyr23 in BPTI, Phe24-Phe25-Tyr26 in insulin (Bchain), and Tyr27-Tyr28-Tyr29 in thermolysin are extended conformations, i.e., EEF, EED, and ECF conformations, respectively, two former sequences are situated in the central region of $\beta$-structure and the last one is situated in the starting point of $\beta$-structure. However, the conformation of Phe261Tyr262-Tyr263 in subtilisin is a non-extended conformation (BBA conformation), i.e., distorted short $\alpha$-helix situated in the turn region of the backbone of subtilisin.

Similar backbone conformations of three tripeptide-sequences taking extended conformations in crystal state are found in the ensemble of minimum-energy conformations of Ac-Phe-Phe-Phe-NHMe. Two EEF conformations similar to Tyr21-Phe22-Tyr23 of BPTI appeared at the 59 and 80th conformations $\left(\Delta E=2.68\right.$ and $2.96 \mathrm{kcal} \mathrm{mol}^{-1}$, respectively) with $\Delta E<3 \mathrm{kcal} \mathrm{mol}^{-1}$. Three EED conformations similar to Phe24-Phe25-Tyr26 of insulin (B-chain) appeared at the 142 , 246 , and 292th conformations $(\Delta E=3.55,4.41$, and $4.63 \mathrm{kcal} \mathrm{mol}^{-1}$, respectively) with $\Delta E<$ $5 \mathrm{kcal} \mathrm{mol}^{-1}$ (no EED conformations with $\Delta E<3 \mathrm{kcal} \mathrm{mol}^{-1}$ ), and 11 analogous type EEE conformations exist with $\Delta E<$ $3 \mathrm{kcal} \mathrm{mol}^{-1}$ (the lowest-energy conformation is $\Delta E=1.24 \mathrm{kcal} \mathrm{mol}^{-1}$ ). Four ECF conformations similar to Tyr27-Tyr28-Tyr29 of thermolysin appeared at the 12, 23, 51, and 70 th conformations $(\Delta E=0.94,1.61,2.56$, and $2.85 \mathrm{kcal} \mathrm{mol}^{-1}$, respectively). The lowestenergy and third conformations are ECC conformations analogous to the ECF one. Phe261-Tyr262-Tyr263 of subtilisin has $\left(\phi_{1}\right.$, $\left.\psi_{1}, \phi_{2}, \psi_{2}, \phi_{3}, \psi_{3}\right)=\left(-158^{\circ},-28^{\circ},-131^{\circ}\right.$, $-18^{\circ},-103^{\circ},-38^{\circ}$ ), i.e., BBA conformation. No BBA conformations have been found in the energy minima of tripeptides ${ }^{23}$ and tetrapeptides. ${ }^{19,24-29}$ The $(\phi, \psi)$ for Phe261 and Tyr262 are those of unstable conformations with intra-residue interactions ${ }^{6}$ and the conformational energy of Ac-Phe-Phe-PheNHMe which has the same dihedral angles as Phe261-Tyr262-Tyr263 of subtilisin is very 
high $\left(\Delta E=44.32 \mathrm{kcal} \mathrm{mol}^{-1}\right)$. Thus BBA conformations are stabilized by medium- and long-range interactions in subtilisn. Based on the about discussion, it is supposed that the tripeptide-sequence composed of Phe and Tyr residues has a tendency to form extended structures with internal interactions of the tripeptide-sequence. That is, the native conformations of these sequences in proteins may be assigned as extended conformations with short-range interactions.

IR absorption spectroscopy and CD measurements showed that oligopeptides composed of Phe residue take non-folded conformations in solution ${ }^{30-32}$ and $\beta$-structures in the solid state, ${ }^{31-33}$ and also that oligopeptides composed of Tyr residue take $\beta$-structures in solutions and the solid state. ${ }^{34}$ These experimental results agree with our calculated results that energetically favorable conformations of Ac-Phe-Phe-Phe-NHMe are extended ones.

\section{REFERENCES}

1. M. Oka and H. A. Scheraga, unpublished data.

2. T. E. Creighton, Nature, 255, 743 (1975).

3. A. Ritonja, B. Meloun, and F. Gubensek, Biochim. Biophys. Acta, 746, 138 (1983).

4. C. Liu, T. Wu and T. Lo, Int. J. Peptide Protein Res., 21, 209 (1983).

5. H. Meirovich and H. A. Scheraga, Macromolecules, 14, 1250 (1981).

6. M. Oka and A. Nakajima, Polym. J., 16, 693 (1984).

7. M. Oka, T. Hayashi, and A. Nakajima, Polym. J., 17, 621 (1985).

8. M. Oka, Y. Baba, A. Kagemoto, and A. Nakajima, Polym. Bull., 21, 377 (1989).

9. M. Oka, Y. Baba, A. Kagemoto, and A. Nakajima, Polym. Bull., 21, 385 (1989).

10. J. P. Vollmer and G. Spach, Biopolymers, 5, 337
(1967).

11. M. Miyake, S. Akita, A. Teramoto, T. Norisuye, and H. Fujita, Biopolymers, 13, 1173 (1974).

12. T. Norisuye, Ph. D. thesis, Osaka University (1973).

13. G. W. Chen, Ph. D. thesis, Kyoto University (1981).

14. IUPAC-IUB Commission on Biological Nomenclature, Biochemistry, 9, 3471 (1970).

15. F. A. Momany, R. F. McGuire, A. W. Burgess, and H. A. Scheraga, J. Phys. Chem., 79, 2361 (1975).

16. M. J. D. Powell, Computer J., 7, 155 (1964).

17. S. S. Zimmerman, M. S. Pottle, G. Nemethy, and H. A. Scheraga, Macromolecules, 10, 1 (1977).

18. S. S. Zimmerman H. A. Scheraga, Biopolymers, 16, 811 (1977).

19. M. Oka, Y. Baba, A. Kagemoto, and A. Nakajima, Polym. J., 21, 585 (1989).

20. P. J. Flory, "Statistical Mechanics of Chain Molecules," John Wiley \& Sons, New York, N.Y., 1969.

21. S. S. Zimmerman and H. A. Scheraga, Biopolymers, 17, 1849 (1978).

22. M. Oka and A. Nakajima, Polym. J., 16, 553 (1984).

23. M. Oka, G. T. Montelione, and H. A. Scheraga, $J$. Am. Chem. Soc., 106, 7959 (1984).

24. J. C. Howard, A. Ali, H. A. Scheraga, and F. A. Momany, Macromolecules, 8, 607 (1975).

25. I. Simon, G. Nemethy, and H. A. Scheraga, Macromolecules, 11, 797 (1978).

26. S. Fitzwater, Z. I. Hodes, and H. A. Scheraga, Macromolecules, 11, 805 (1978).

27. J. S. Anderson and H. A. Scheraga, Macromolecules, 11, 812 (1978).

28. S. S. Zimmerman, R. Baum, and H. A. Scheraga, Int. J. Peptide Protein Res., 19, 143 (1982).

29. E. Lee, G. Nemethy, H. A. Scheraga, and V. S. Anantharayanan, Biopolymers, 23, 1193 (1984).

30. M. H. Baron, C. de Loze, C. Toniolo, and G. D. Fasman, Biopolymers, 17, 2225 (1978).

31. M. Palumbo, S. DaRin, G. M. Bonora, and C. Toniolo, Makromol. Chem., 177, 1477 (1976).

32. C. Toniolo, G. M. Bonora, M. Palumbo, E. Peggion, and E. S. Stevens, Biopolymers, 17, 1713 (1978).

33. M. H. Baron, C. de Loze, C. Toniolo, and G. D. Fasman, Biopolymers, 18, 411 (1979).

34. G. M. Bonora, V. Moretto, C. Toniolo, H. Anzinger, and M. Mutter, Int. J. Peptide Protein Res., 21, 336 (1983). 